Computer Standards and Interfaces, Vol. 26, pp. 51-56, 2004.

\title{
FOUR PARAMETER FITTING OF SINE WAVE TESTING RESULT: ITERATION AND CONVERGENCE
}

\author{
TAMÁS ZOLTÁN BILAU, TAMÁS MEGYERI, ATTILA SÁRHEGYI, \\ JÁNOS MÁRKUS, and ISTVÁN KOLLÁR \\ Budapest University of Technology and Economics \\ Department of Measurement and Information Systems \\ Budapest, Hungary, H-1521, P. O. Box 91. \\ Phone: +36 1 463-1774, fax: +36 1 463-4112, e-mail:kollar@mit.bme.hu
}

\begin{abstract}
Small improvements to the iteration procedure of the IEEE Standard 1241-2001 are suggested, and extension of the standard MATLAB program implementing the sine wave test is discussed. The program is compatible with the LabView program already announced, and in other working modes offers extensions, too.
\end{abstract}

Keywords: IEEE-STD-1241, ADC testing, sine wave method, MATLAB, four-parameter method

\section{Introduction}

Unfortunately, no standard can deal with all details of the associated calculation procedures. This is especially true for iterative procedures. Starting values, stop criteria, numerical details can differ from implementation to implementation, and can cause different results in different implementations even when processing the same data. This causes special problems. The IEEE standard on ADC testing [1] defines the way how to make a reasonable sine fit to the measured data in a sine wave test. However, the so-called four parameter fitting, described in detail in this standard (Section 4.1.4.3), is not fully defined. This procedure is of iterative nature, therefore circumstances like starting values, way of calculation, stop criteria, etc. need to be exactly defined. While the recursive steps are precisely described in the standard the stop criterion of the iteration is not 
specified at all, and the setting of the starting value is not uniquely specified. However, we think that internationally reproducible results can only be achieved if these details are uniquely defined. Therefore, we need to move in the direction of more precisely fixing these algorithms - either in the standard, or is some associated document.

\section{Discussion}

In the mathematical literature, these details of iterative numerical methods are extensively discussed. Therefore, it is possible to use these to exactly define the details of our algorithm.

\subsection{Starting values}

Setting of the starting value is described in the standard as "Make an initial estimate of the angular frequency $\omega_{0}$ of the recorded data. The frequency may be estimated by using a DFT (either on the full record or a portion of it), or by counting zero crossings, or simply by using the applied input frequency." While this is correct from scientific viewpoint, leaving a choice to the user can hinder international reproducibility even on the same data. As [3] points out, for short records even convergence can change with the setting of the starting values, especially when the phase of the sine wave takes certain values. Also, convergence speed may depend on proper setting of the starting values. By default, the procedure needs to have at least one default way of calculation. The cost function depend on the number of samples too Fig.1. The length of record is bigger the valley of global minima is become less wide. But the accuracy of DFT is grow and the starting value stay around the global minima.

\subsection{Calculation method}

The standard number representation for scientific calculations is IEEE double precision, like in MATLAB. However, even using this, the expression (4.1.4.3.6) is numerically inefficient, and imprecise. Instead of the calculation of

$$
x_{0}=\left(D_{i}^{T} D_{i}\right)^{-1}\left(D_{i}^{T} y\right),
$$

one needs to use rather matrix factorization algorithms to solve $y=D_{i} x_{i}$. [7] The result is theoretically the same, however, in extreme cases the explicit solution may give erroneous results while the numerical solution still works.

\subsection{Stop criterion}

An iterative algorithm needs to perform a finite number of iterations. The problem is in general that the number of necessary iterations depends on the nature of data, so it cannot be given in advance. If we observe the change in the cost function, and the limit of change is set too high, the error will still be 
too large at the end, while if the limit of change is too low, we waste our time on useless iterations.

\section{Improvement of the starting values}

Although IEEE1241 [1] requires that at least 4 periods of the sine wave should be sampled, and this is usually enough to avoid getting into local minima, it can be made possible that the algorithm works well even when the record length is less than 4 periods. The key is to find a good starting frequency value.

By using DFT, we know the frequency with accuracy $\frac{1}{2 M \Delta t}$, that is, half of the bin width, with $\Delta t$ being the sampling time, and $M$ the number of samples. In order to improve frequency accuracy, we can use interpolated DFT (IpDFT). $[8]$.

$$
f=(L+\delta) \Delta f=\lambda \Delta f \quad 0 \leq \delta<1
$$

The algorithm is as follows. The DFT of the time series is taken, and the maximum and its larger neighbor are selected, where $0 \leq L<M$.

$$
\begin{gathered}
X(L)=U_{L}+j V_{L} \\
X(L+1)=U_{L+1}+j V_{L+1}
\end{gathered}
$$

Then,

$$
\lambda=\frac{\arccos \left(\frac{Z_{2} \cos (n(L+1))-Z_{1} \cos (n L)}{Z_{2}-Z_{1}}\right)}{n}
$$

with

$$
\begin{gathered}
Z_{1}=V_{L}\left(\frac{K_{o p t}-\cos (n L)}{\sin (n L)}\right)+U_{L} \\
Z_{2}=V_{L+1}\left(\frac{K_{o p t}-\cos (n(L+1))}{\sin (n(L+1))}\right)+U_{L+1} \\
K_{o p t}=\frac{\left((\sin (n L))\left(V_{L+1}-V_{L}\right)+(\cos (n L))\left(U_{L}+U_{L+1}\right)\right)}{U_{L+1}-U_{L}}
\end{gathered}
$$

where $n=\frac{2 \pi}{M}$. By using IpDFT to determine the start frequency, a significantly better result can be found than by DTF, as shown in Fig.2. By using $f_{1}=0.020773 f_{s}$ a sine frequency and $n=70$ points, the four parameter fit to the sine wave

$$
y=\sin (2 \pi \cdot 0.020773 \cdot[1: 70]) ;
$$

is bad when using DFT to estimate initial frequency, but when using IpDFT a good result is determined. With DFT we get to a local minimum at: $f_{0}=$ 
$0.042293 f_{s}$, while by IpDFT we get to the global minimum with the true $f_{1}$. This is illustrated by the program, non-convergence in compatible mode, and convergence in standard mode (with IpFFT). These data are available for comparison on the Internet [14].

In general, it is true that when having more than 4 periods of a sine wave, it can be recommended but it is not necessary to use IpDFT. If using IpDFT, it's somewhat more probable that we get to the global minimum.

\section{Analysis of frequency precision}

An interesting question in four parameter fitting is the following: how many iterations are needed, and how accurately the result need to be displayed (nonsignificant digits should not be shown). The measured sine wave is imprecise, because of observation noise, quantizaton noise, parameter inaccuracies, etc. In this case the cost function analysis would be too difficult, so it needs simplification. We analyze here only the effect of frequency inaccuracy.

The four parameter least squares fit to a sine wave minimizes the following sum (cost function) of the squared differences:

$$
e=\sum_{n=1}^{M}\left(y_{n}-A \cos \left(\omega t_{n}\right)-B \sin \left(\omega t_{n}\right)-C\right)^{2}
$$

Assume that the errors stem only from the inaccurately given frequency:

$$
e=\sum_{n=1}^{M} r^{2}=\sum_{n=1}^{M}\left(A \sin \left(\omega t_{n}\right)-A \sin (\omega+\Delta \omega) t_{n}\right)^{2}
$$

Utilize the next relationship:

$$
\int_{0}^{k T} r^{2} d t=\sum_{n=1}^{M} r^{2} \Delta t \text { where } \Delta t=\frac{k T}{M}
$$

Sufficiently close to the minimum, we expect that the cost function can be well approximated by a quadratic form:

$$
e=\frac{1}{\Delta t} \int_{0}^{k T}(A \sin (\omega t)-A \sin (\omega+\Delta \omega) \cdot t)^{2} d t \approx \frac{2(A k \pi)^{2} M}{3} \cdot\left(\frac{\Delta \omega}{\omega}\right)^{2}
$$

The rms error is:

$$
e_{r m s}=\sqrt{\frac{1}{M} \sum_{n=1}^{M} r_{n}^{2}}=\sqrt{\frac{2}{3}} \cdot A k \pi\left(\frac{\Delta \omega}{\omega}\right)
$$

If we prescribe that the rms error is smaller than half of the standard deviation of the quantizer error: 


$$
e_{r m s}<0.5 \sigma_{q}=0.5 \cdot \frac{\left(\frac{2 A}{2^{N}}\right)}{\sqrt{12}},
$$

where $N$ is the number of digitized bits, we obtain:

$$
\frac{\Delta \omega}{\omega}<\frac{\sqrt{2}}{k \pi 2^{N+2}}
$$

Fig.3 illustrates this relationship.

Some numerical examples:

- if the number of bits is $N=8, \frac{\Delta \omega}{\omega}<4.4 \cdot 10^{-4}$, so we need 5 digits,

- if the number of bits is $N=20, \frac{\Delta \omega}{\omega}<1.07 \cdot 10^{-7}$, so we need 8 digits.

\section{Cramér-Rao bound}

In the previous section, we analyzed the effect of frequency inaccuracy on the cost function. We arrived at the conclusion that the relative frequency error need not decrease to zero, because on the one hand, we would waste time on useless iterations. There is a lower bound on the variance of the estimated parameters, so we cannot get any better estimate. This lower bound on the variance is the so-called Cramér-Rao bound (CRB).

In the distortionless case this lower bound of the conditional covariance matrix can be calculated from [9]

$$
\operatorname{cov}[\widetilde{a}, \widetilde{a} \mid a]=E\left\{(\widetilde{\alpha}-a)(\widetilde{\alpha}-a)^{T} \mid a\right\} \geq J^{-1}
$$

where $\mathrm{J}$ is the Fisher information matrix.

$$
J_{i j}=E\left\{\frac{\partial \ln f_{z \mid a}(z \mid a)}{\partial a_{i}} \cdot \frac{\partial \ln f_{z \mid a}(z \mid a)}{\partial a_{j}}\right\}=-E\left\{\frac{\partial^{2} \ln _{z \mid a}(z \mid a)}{\partial a_{i} \partial a_{j}}\right\}
$$

For uniform sampling $t_{k}=\frac{k}{f_{s}}$ with $f_{s}$ being the sampling frequency, an approximation of the CRB of the absolute frequency $\widehat{f}$ and large $N$ is [10]

$$
\begin{aligned}
\operatorname{CRB}(\widehat{f}) & \approx\left(\frac{f_{s}}{2 \pi}\right)^{2} \cdot \frac{2 \sigma^{2}}{\left(A^{2}+B^{2}\right)} \cdot \frac{12}{N\left(N^{2}-1\right)}= \\
& =\left(\frac{f_{s}}{2 \pi}\right)^{2} \cdot \frac{12}{S N R \cdot N \cdot\left(N^{2}-1\right)}
\end{aligned}
$$

where $S N R$ denotes the signal-to-noise ratio, that is, $S N R=\frac{\left(A^{2}+B^{2}\right)}{2 \sigma^{2}}$. The asymptotic result (19) only depends on the $S N R$. In particular, it is independent of the absolute frequency and the initial phase of the sine wave. 


\section{Consequences of precision to the program}

We implemented our results (starting values, numerical methods and stop criteria) in the standard MATLAB software package [13]. In addition to the working mode which is fully compatible with the present LABVIEW one [11], in other working modes we made use of the above results. These do not significantly change the working of the program, but allow improved usability.

When we know the required precision of certain quantities, we can use this information in two ways.

- the stop criterion can be set according to this,

- the displaying of the calculated values can be shown up to the last significant digit only.

\section{Conclusions}

Extensions have been suggested for the definition of the iterative procedure, for starting values (based on the Interpolated FFT), numerical methods (based on matrix factorizations), and stop criteria (we suggest making use of the bit number of the ADC to precisely set the limits). These can be used as default solutions, and extensions which work even more reliably under certain circumstances than previously define.

In addition, we have developed expressions for the variance of the parameter estimation procedures, and their effect to the effective bit number. The program is available via the internet [13].

\section{References}

[1] IEEE TC-10, IEEE Std 1241-2001, Standard for Terminology and Test Methods for Analog-to-Digital Converters.

[2] IEEE TC-10, IEEE Std 1057-94 - IEEE Standard for Digitizing Wave-form Recorders.

[3] M. Fonseca da Silva, A. Cruz Serra, 'Improving Convergence Of Sine Fitting Algorithms', Proc. 6 Euro Workshop on ADC Modelling and Testing, September 13-14, 2001, Lisbon, Portugal. pp. 121-124.

[4] J. Márkus and I. Kollár, "Standard environment for the sine wave test of ADC's," Submitted to the Measurement Journal of IMEKO, February, 2002. vol 31. pp. 261-69

[5] N. Giaquinto and A. Trotta, "Fast and accurate ADC testing via an enhanced sine wave fitting algorithm," IEEE Trans. on Instrumentation and Measurement, vol. 46, no. 4, pp. 1020-1024, August 1997. 
[6] P. Arpaia et al., "ADC testing based on IEEE 1057-94 standard - some critical notes," in Proc. of the 17th IEEE Instr. and Meas. Technology Conference, IMTC/2000, Baltimore, Maryland USA, May 2000, vol. 1, pp. 119-124.

[7] Press, W. H., B. P. Flannery, S. A. Teukolsky and W. T. Vetterling, Numerical Recipes: The Art of Scientific Computing. Cambridge University Press, Cambridge, 1986.

[8] Schoukens, J., R. Pintelon and H. Van hamme, 'The Interpolated Fast Fourier Transform: A Comparative Study,' IEEE Trans. on Instrumentation and Measurement, IM-41, No. 2, April 1992, pp.226-32.

[9] Pieter Eykhoff: System Identification. Parameter and State Estiamtion.

[10] Peter Händel: Properties of the IEEE-STD-1057 Four-Parameter Sine Wave Fit Algorithm. IEEE Transactions on Instrumentation and Measurement, Vol. 49, No. 6, DECEMBER 2000.

[11] J. J. Blair, "Sine-fitting software for IEEE standards 1057 and 1241," in Proc. of the 16th IEEE Instr. and Meas. Technology Conference, IMTC/99, Venice, Italy, May 1999, vol. 3, pp.1504-1506.

[12] J. Márkus and I. Kollár, 'A MATLAB Tool to Execute IEEE-STD 1241' Proc. IEEE Instrumentation and Measurement Technology Conference, IMTC/2001, Budapest, Hungary, May 21-23, 2001. pp.1847-52.

[13] J. Márkus, 'ADC Test Data Evaluation Program for Matlab' Home Page URL:http://www.mit.bme.hu/services/ieee/ADC-test/.

[14] Test data. URL: http://www.mit.bme.hu/services/ieee/ADC-test/data/. 


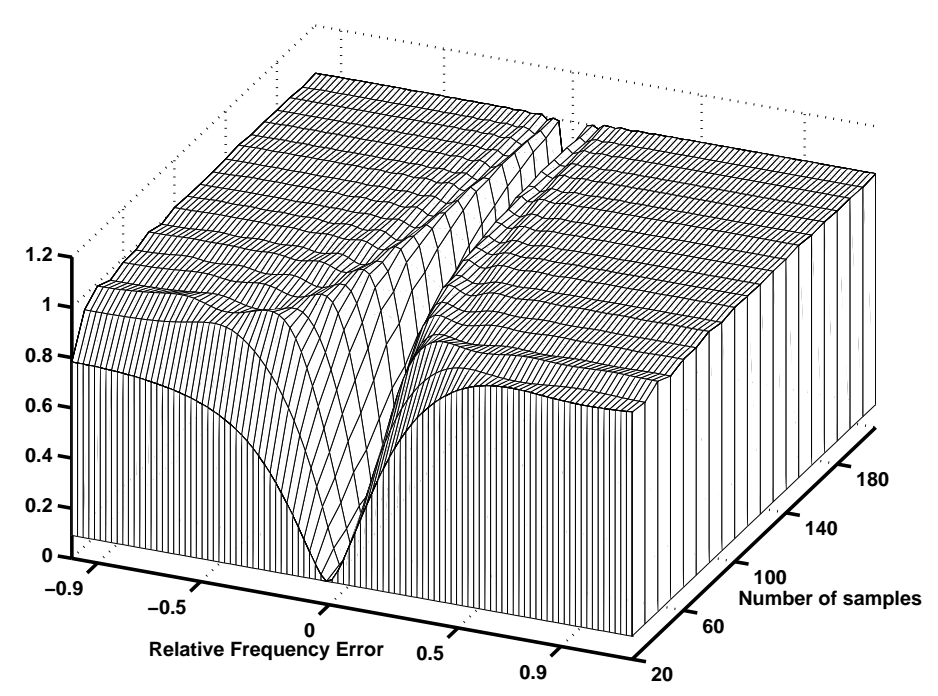

Fig. 1: The rms error of the three parameter algorithm in LSB as a function of the relative frequency error and the number of bits. 


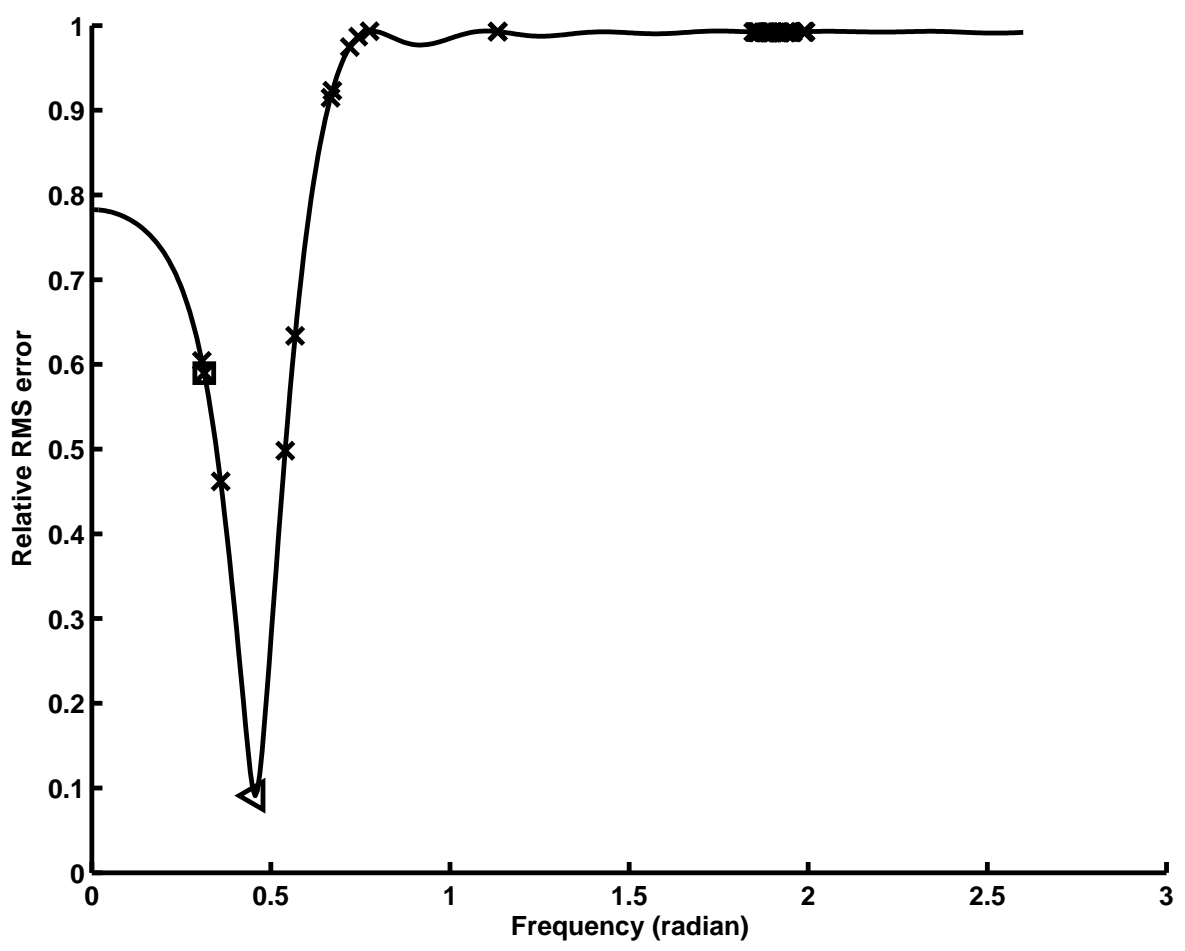

Fig. 2: The rms error of the three parameter algorithm in LSB as a function of the applied frequency value show as related to the nominal value $f_{1}=0.020773 f_{s}, M=70$ points, no noise. $\square$ The frequency estimated by DFT, and $\triangleleft$ The frequency estimated by IpDFT. 


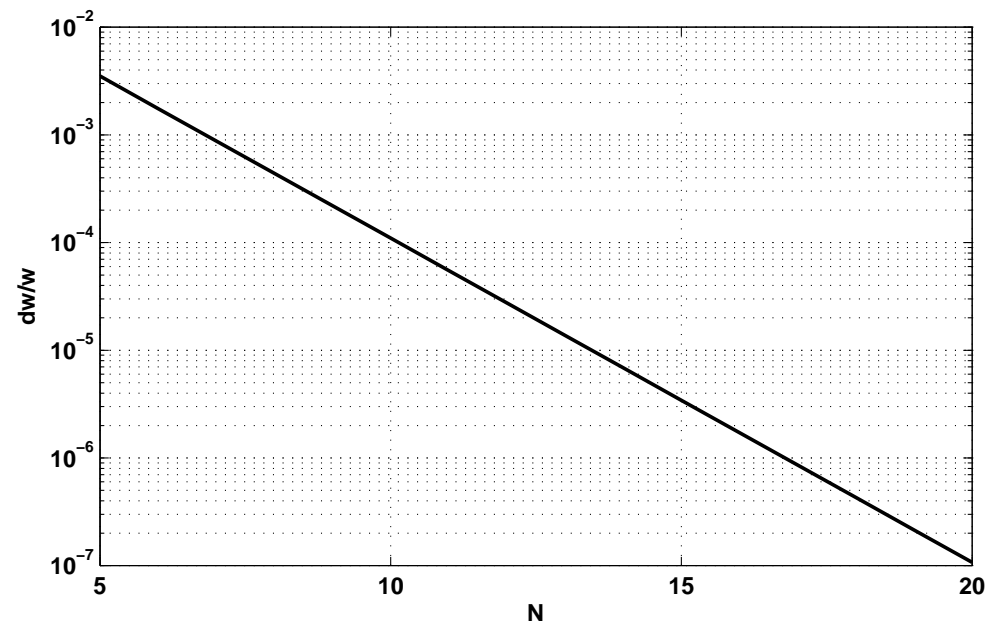

Fig. 3: Allowable relative frequency error as a function of the number of bits. 